\title{
Llegaron en sus canoa: innovación gramatical en el español de la Amazonía peruana
}

Llegaron en sus canoa: Grammatical Innovation in Peruvian Amazonian Spanish

\author{
Rosa Vallejos \\ University of New Mexico, Albuquerque, Nuevo México, Estados Unidos \\ Contacto: rvallejos@unm.edu \\ https://orcid.org/0000-0001-7282-7611
}

\begin{abstract}
Resumen
Este estudio investiga las motivaciones cognitivas y funcionales detrás del surgimiento de una construcción posesiva innovadora en el español de la Amazonía peruana. En esta variedad, el núcleo de un sintagma nominal no concuerda necesariamente en número con su modificador, cuando este es un pronombre posesivo. Así, sintagmas del tipo sus canoa son comunes en la Amazonía. Un estudio previo encontró que las probabilidades de uso de este patrón innovador aumentan cuando el pronombre hace referencia anafórica a múltiples individuos. El presente estudio examina exclusivamente contextos con poseedores múltiples para identificar los parámetros que rigen la alternancia entre patrones concordantes y discordantes. Las variables que juegan un rol son el género del hablante y la animacidad del poseedor y el poseído. El uso del patrón innovador es ligeramente más frecuente entre las mujeres, sobre todo cuando el pronombre posesivo hace referencia a múltiples entidades altas en animacidad y el sustantivo a una entidad baja en animacidad.
\end{abstract}

Palabras claves: Indigenismo; Español amazónico; Posesión; Animacidad; Variación morfosintáctica.

\begin{abstract}
This study investigates the cognitive and functional motivations behind the emergence of an innovative construction in Peruvian Amazonian Spanish. In this variety, the head of a noun phrase does not necessarily agree in number with its modifier, when this is a possessive pronoun. Thus, noun phrases with number mismatch, such as sus canoa 'their canoe,' are common. A previous study found that the probabilities of using this innovative pattern increase when the pronoun makes anaphoric reference to multiple individuals. The present study examines exclusively contexts with multiple possessors to identify the parameters behind the alternation between concordant and discordant patterns. The variables that play a role are the gender of the speaker and the animacy
\end{abstract}


of the possessor and the possessed. The use of the innovative pattern is slightly more frequent among women, and when the possessive pronoun refers to multiple entities high in animacy and the possessed noun to an entity low in animacy.

Keywords: Amazonian Spanish; Possession; Animacy; Morphosyntactic variation.

Recibido: 30.09 .18

Aceptado: 08.03.19

\section{Introducción ${ }^{1}$}

Este estudio emplea un enfoque cuantitativo para elucidar los detalles subyacentes al uso de una construcción posesiva innovadora en el español hablado en la región Loreto, Perú ${ }^{2}$. Según datos del Instituto Nacional de Estadística e Informática (INEI, 2017), Loreto tiene la mayor población indígena amazónica en el país. Esta alberga una gran diversidad lingüística, incluyendo cerca de 23 lenguas distribuidas en diez familias lingüísticas (Tupí-Guaraní, Záparo, Tucano, Arawak, Jíbaro, Cahuapana, Pano, Peba-Yagua, Bora, Witoto), además de aproximadamente seis lenguas aisladas (kandozi, tikuna, yagua, taushiro, urarina, cholón) (Queixalós, 2009; Solís Fonseca, 2009). Sin embargo, los hablantes de las lenguas originarias representan un porcentaje pequeño de la población total de la región. En el último censo, el 9,8\% de la población loretana se autoidentificó como perteneciente a un pueblo indígena; sin embargo, solo el 6,4\% reportó haber aprendido a hablar en una lengua indígena, y el 89,97\% declaró que lo hizo en español (INEI, 2017). La composición sociolingüística de Loreto es, entonces, bastante compleja.

En español canónico, el núcleo del sintagma nominal determina el número y el género de todos sus modificadores. Sin embargo, en el español de la Amazonía peruana en construcciones del tipo [PRO N], el número del pronombre posesivo no coincide necesariamente con el de su núcleo nominal. Ello da como resultado las siguientes posibilidades: concordancia singular (su canoa), concordancia plural (sus canoas), y discordancia plural/singular (sus canoa). La cuarta posibilidad lógica (su canoas) no ha sido documentada (Vallejos, 2014). La construcción innovadora se distancia de manera excepcional de patrones 
canónicos de concordancia al interior del sintagma nominal, y, hasta donde tenemos entendido, no ha sido reportada para ninguna otra variedad de español. Considérese el siguiente ejemplo identificado en el corpus:

\section{(1) los padres poco a poco llegaron en sus canoa}

Nótese que el pronombre posesivo sus hace referencia a varios individuos: los padres de familia; pero también existen varios elementos poseídos: las canoas. En este contexto, otras posibles expresiones que el entrevistado pudo haber empleado son: la concordancia plural - los padres poco a poco llegaron en sus canoas; o incluso la concordancia singular - los padres poco a poco llegaron en su canoa.

Vallejos (en evaluación) investigó la alternancia entre estas tres construcciones posesivas (concordancia singular, concordancia plural y discordancia plural/singular) y el hallazgo más importante fue que la construcción innovadora es un patrón morfosintáctico omnipresente en el español amazónico peruano (EAP). Su uso trasciende los diferentes estratos sociales, grupos generacionales, ocupaciones, así como ámbitos tanto rurales como urbanos, $\mathrm{y}$, actualmente, es ampliamente usado en las redes sociales. Sin embargo, ciertos factores favorecen el uso del patrón innovador por sobre las opciones conservadoras. El nuevo patrón impone ciertas restricciones al referente del pronombre posesivo. La construcción innovadora compite con las construcciones conservadoras cuando el poseedor hace referencia a varias entidades. En otras palabras, el pronombre posesivo concuerda en número con la entidad a la que hace referencia anafórica, no necesariamente con la cabeza del sintagma nominal. Crucialmente, la construcción discordante no es usada en todos los contextos en los que están involucrados múltiples poseedores; solo en algunos de ellos.

El presente estudio se basa en hallazgos previos (Vallejos, en evaluación) para profundizar en los posibles factores que motivan el uso de la opción innovadora. Así, las instancias de [PRO N] seleccionadas para este estudio corresponden exclusivamente a contextos comunicativos que involucran 
a varios poseedores. Las preguntas que guían este estudio son las siguientes: ¿Cuál es la distribución global de la construcción innovadora en relación con las conservadoras? ¿Qué factores sociales y/o lingüísticos (des)favorecen su uso? ¿La construcción innovadora está asociada con algún subtipo de posesión?

Estas preguntas se exploran en un corpus compuesto de entrevistas sociolingüísticas recogidas de 21 hablantes monolingües. Los entrevistados son originarios de comunidades kukama y de la ciudad de Iquitos (los detalles de la muestra se explican en §3.1). Las variables sociales codificadas son el lugar de origen y el género de los hablantes. Las variables lingüísticas incluyen animacidad del poseedor y el poseído, cuantificación del poseedor y el poseído, y subtipos de relaciones posesivas. Los resultados más importantes son los siguientes: (i) el lugar de origen de los hablantes y los subtipos de posesión no juegan un papel en la discordancia de número; (ii) el referente del pronombre posesivo tiende a ubicarse en la posición más elevada de la jerarquía de animacidad, y el referente del núcleo del sintagma nominal tiende a ubicarse en la posición más baja de la jerarquía de animacidad. Este estudio demuestra que, en español Amazónico, el núcleo del sintagma nominal compite con el referente del pronombre posesivo para controlar la concordancia de número. En un contexto en el que el sustantivo hace referencia a una entidad baja en animacidad, pero el pronombre se refiere a múltiples entidades altas en animacidad, "gana" el referente del pronombre, lo que tiene como resultado la discordancia de número al interior del sintagma nominal. En suma, proponemos que funciones cognitivas generales que trascienden el lenguaje — como la categorización de entidades, la atención preferencial a entidades animadas - juegan un rol fundamental en el surgimiento del patrón innovador. Este estudio proporciona evidencias concretas sobre el rol que cumplen los factores extralingüísticos en el uso del lenguaje y, ultimadamente, contribuye a una visión de la gramática como un fenómeno dinámico y emergente.

\section{Posesión}

En esta sección, presentamos brevemente el enfoque sobre la posesión que se sigue en este estudio, así como la literatura existente sobre la posesión en 
el español amazónico. Los detalles de cada variable incluida en este estudio se ofrecen en $\$ 3.4$.

\subsection{Posesión nominal}

La posesión, a nivel conceptual, es un fenómeno universal (Heine, 1997; Stassen, 2009). El significado de una construcción posesiva en general implica tres elementos principales: dos entidades —el poseedor y el poseído- y una relación entre ellos. Una distinción básica en la expresión gramatical de la posesión es la que existe entre construcciones atributivas (ej. su casa) y predicativas (ej. tiene una casa). Mientras que las construcciones atributivas presuponen la posesión, las construcciones predicadas establecen la posesión. En el lado formal, la construcción atributiva implica modificadores de sustantivos al interior de sintagmas nominales, mientras que las construcciones predicativas implican cláusulas. Las construcciones atributivas y las construcciones predicativas pueden tener funciones especializadas en las lenguas del mundo ${ }^{3}$. Este estudio está dedicado exclusivamente a la posesión nominal expresada en construcciones del tipo [PRO N].

Un punto interesante es que la expresión lingüística de la posesión tiende a renovarse con relativa rapidez (Heine, 1997, p. 141). Esto significa que la posesión es una de aquellas áreas de la gramática particularmente dinámicas y sensibles al cambio. Por ejemplo, el español y el portugués han renovado su principal construcción posesiva desde el mihi est del latín, hasta el esquema del tipo tener/ter, pasando por el esquema del tipo habeo. El patrón posesivo innovador examinado aquí podría considerarse un ejemplo adicional de esta tendencia dinámica en el dominio de la posesión.

\subsection{Posesión en el EAP}

Como se indicó anteriormente, los estudios detallados sobre el EAP son escasos, particularmente en el área de la posesión ${ }^{4}$. El patrón de discordancia examinado aquí ha sido mencionado brevemente en la literatura. Barraza de la Cruz (1998, p. 56) indica que en estas construcciones “[...] la pluralidad está entendida con 
relación al poseedor, y no al poseído. Los poseedores son varios, ellos o ustedes. La pluralidad de lo planteado es definido por el contexto”. Según Ramírez,

[...] en el plural de la posesión, los posesivos se usan correctamente pero el sustantivo poseído se inmoviliza en singular, con lo que la frase posesiva mantiene una discordancia de número entre posesivo y sustantivo: mis silla; tus cuaderno; mis ropa; sus padre; digan sus nombre son formas plurales en el dialecto amazónico. (2003, p. 36).

Nótese que según Barraza de la Cruz la discordancia de número ocurre cuando el pronombre hace referencia a la segunda y la tercera persona plural, y, de acuerdo con Ramírez, con la primera, segunda y tercera persona, tanto singular como plural. Contrario a lo que sostienen estos autores, Vallejos (2014) reporta que, en el corpus Conversaciones en Loreto (véase detalles en §3), el pronombre posesivo en la construcción innovadora nunca hace referencia a la primera o segunda persona. Es decir, el patrón innovador involucra exclusivamente a la tercera persona plural ${ }^{5}$.

Un estudio que se ha centrado en la posesión nominal en el EAP es el de Vallejos (en evaluación). El patrón innovador no ocurre al azar, sino que la alternancia entre las tres construcciones (concordancia singular, concordancia plural y discordancia plural/singular) sigue ciertas restricciones sobre los poseedores. Un análisis de 656 instancias de la construcción [PRO N] extraídas del corpus Conversaciones en Loreto revela que el $24 \%$ de ellas (157/656) involucra múltiples poseedores. Asimismo, el $27 \%$ de las instancias con poseedores múltiples (42/157) están expresadas a través de la construcción innovadora. En otras palabras, en aproximadamente el $73 \%$ de los casos el pronombre posesivo concuerda con el núcleo del sintagma nominal, siguiendo patrones canónicos. Entonces, en el EAP un nuevo candidato está emergiendo como referente del pronombre posesivo: su anáfora (el poseedor). Este elemento externo al sintagma nominal compite con la cabeza sintáctica del sintagma (el poseído) por el control de la concordancia.

El presente estudio evalúa los resultados reportados previamente en un corpus más amplio. A diferencia de Vallejos (en evaluación), este se enfoca 
exclusivamente en aquellos contextos donde están involucrados múltiples poseedores; el elemento poseído expresado en la cabeza del sintagma nominal puede hacer referencia a una o varias entidades. La metodología de este estudio se explica a continuación.

\section{Metodología}

Para explorar las preguntas de investigación mencionadas en la introducción, se examinó muestras de español amazónico de 21 hablantes monolingües. Estas muestras son parte del corpus Conversaciones en Loreto que, por ahora, consta de aproximadamente 40 horas de entrevistas a un total de 52 hablantes. Las entrevistas examinadas en este estudio se realizaron entre 2011 y 2016, y su duración oscila de 35 a 65 minutos. Cada entrevista está diseñada para que los participantes guíen la conversación según los temas de su preferencia. El primer tema fue presentado por la entrevistadora (ej. los últimos acontecimientos en el lugar, su experiencia personal en la escuela, actividades preferidas, etc.). Las entrevistas han sido transcritas en unidades entonacionales con el programa Transcriber 1.5.2 (Barras, 2000).

\subsection{Muestra}

El español hablado en la región amazónica tiende a ser retratado como una variedad vernácula de transición, usada por hablantes para quienes el español es un idioma no nativo (Solís Fonseca, 2009, p. 326), fundamentalmente rural e indígena (Lipski, 1994, pp. 322-327). No obstante, como se demuestra en Vallejos (2014), la mayoría de las características consideradas representativas de esta variedad pueden identificarse entre los hablantes de todo el espectro social. Dada la diversidad lingüística y cultural de la región, así como su compleja historia, categorías como rural y urbano no son necesariamente relevantes para estudiar el español amazónico. Sería desatinado, por ejemplo, tratar de hacer generalizaciones para el área "rural" de todo Loreto a partir de hallazgos en ciertas comunidades. Creemos que es importante hacer un seguimiento cuidadoso de los fenómenos lingüísticos en comunidades con una historia y un perfil sociolingüístico específico para luego comparar estos resultados con observaciones en comunidades con otro 
tipo de sustrato, y con lo que encontramos en centros urbanos como Iquitos. Esa es la aproximación que seguimos aquí.

Para el presente estudio, se construyó una muestra equilibrada en función del lugar de procedencia, género y edad. La tabla 1 muestra la información demográfica de los hablantes. Los participantes tienen ocupaciones varias, pero todos han tenido acceso a más de 10 años de educación formal ${ }^{6}$.

Tabla 1. Hablantes entrevistados

\begin{tabular}{|c|c|c|c|c|}
\hline Procedencia & Hablante & Género & Edad & Ocupación \\
\hline \multirow{11}{*}{$\begin{array}{l}\text { Comunidades } \\
\text { kukama }\end{array}$} & COM-1 & $\mathrm{F}$ & 17 & estudiante de secundaria \\
\hline & COM-2 & $\mathrm{F}$ & 19 & estudiante universitario \\
\hline & COM-3 & $\mathrm{F}$ & 30 & profesora \\
\hline & COM-4 & $\mathrm{F}$ & 45 & negociante \\
\hline & COM-5 & $\mathrm{F}$ & 53 & ama de casa \\
\hline & COM-6 & M & 21 & estudiante de pedagógico \\
\hline & COM-7 & M & 21 & estudiante de pedagógico \\
\hline & COM-8 & $M$ & 24 & estudiante de pedagógico \\
\hline & COM-9 & M & 33 & profesor \\
\hline & COM-10 & M & 35 & agricultor \\
\hline & COM-11 & $M$ & 39 & profesor \\
\hline \multirow{10}{*}{ Iquitos } & IQT-1 & $F$ & 21 & estudiante universitario \\
\hline & IQT-2 & $\mathrm{F}$ & 35 & ama de casa \\
\hline & IQT-3 & $\mathrm{F}$ & 37 & enfermera \\
\hline & IQT-4 & $\mathrm{F}$ & 39 & profesora \\
\hline & IQT-5 & $\mathrm{F}$ & 51 & empleada pública \\
\hline & IQT-6 & M & 17 & estudiante de secundaria \\
\hline & IQT-7 & M & 26 & artista plástico \\
\hline & IQT-8 & M & 39 & empleado público \\
\hline & IQT-9 & M & 41 & empleado público \\
\hline & IQT-10 & M & 29 & profesor \\
\hline
\end{tabular}

Elaboración propia. 
El primer grupo de hablantes proviene de ocho comunidades kukama ubicadas a lo largo de los ríos Samiria, Marañón y sus afluentes, como se indica en el mapa 1. Las comunidades son Payorote, Solterito, San Ramón, Dos de Mayo, Nueva Fortuna, Parinari, San Martín de Tipishca y Nuevo Arica. La distancia entre estas comunidades e Iquitos varía entre 18 y 24 horas en lancha. En las comunidades seleccionadas, el español ha desplazado a la lengua kukama hace unas cinco décadas. Los hablantes de esta lengua ancestral son escasos, y ciertamente ninguno de ellos es monolingüe en kukama (Vallejos, 2016). Al momento de las entrevistas, las comunidades incluidas en este estudio tenían acceso a la radio, pero no a la televisión ni al internet. Estas comunidades cuentan con escuelas de educación primaria, pero solo cuatro de ellas — San Martín del Tipishca, Parinari, San Ramón y Nueva Fortuna - ofrecen educación secundaria. Por lo tanto, algunos participantes reportan que asistieron a la escuela secundaria en una comunidad vecina. Algunos kukamas suelen viajar regularmente a los centros poblados y ciudades más cercanas, como Nauta e Iquitos (véase mapa 1).

El segundo grupo de hablantes proviene de Iquitos, el puerto más importante del río Amazonas dentro del territorio peruano. Iquitos es la ciudad más grande de la Amazonía del Perú con una población total de 159.023 (INEI, 2017). La ciudad está rodeada por distritos como Belén, Punchana y San Juan; incluyendo a los habitantes de estos distritos, la población total suma 406.340 (INEI, 2017). En Iquitos, el 99\% de la población se declara hispanohablante (INEI, 2017). Y aunque Iquitos atrae a miembros de pueblos indígenas que se ubican cerca de la ciudad, solo el 0,2\% declara conocer otro idioma local. Es común que los jóvenes de las comunidades migren a Iquitos para trabajar y estudiar. Debido a que los kukamas hablan español, es relativamente fácil para ellos integrarse en la vida de Iquitos en comparación con miembros de otros grupos. 
Mapa 1. Ubicación de las comunidades kukamas y la ciudad de Iquitos

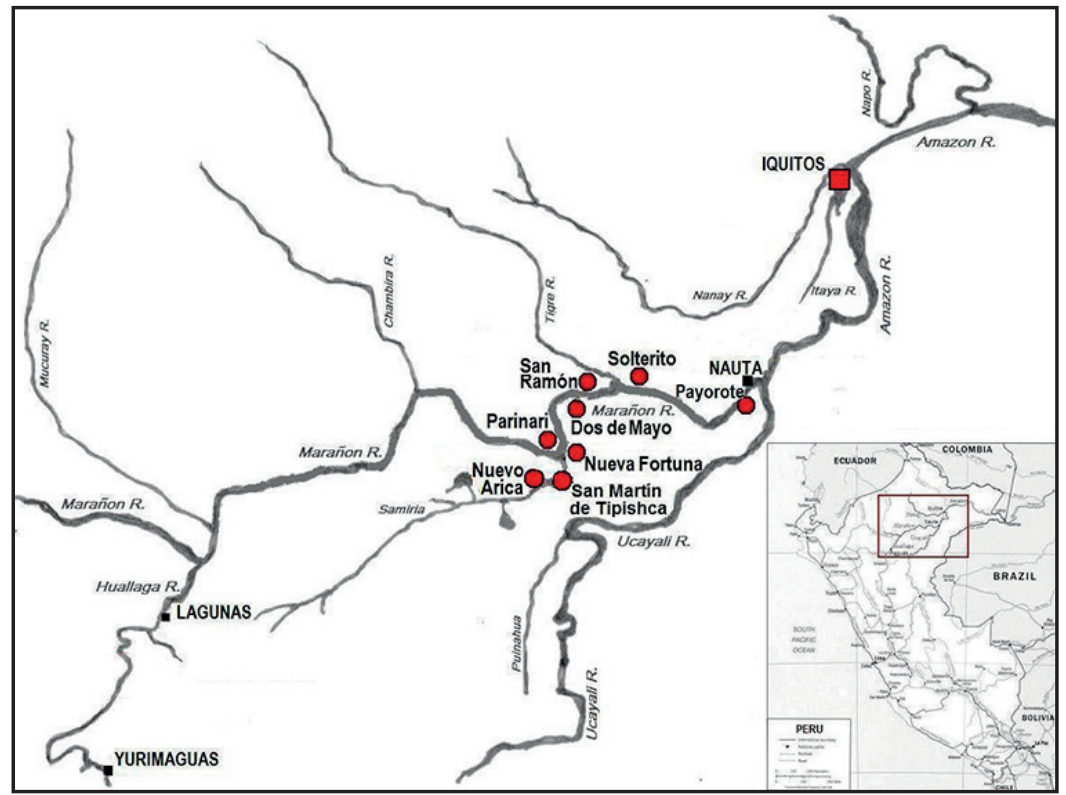

Fuente: Vallejos, 2014, p. 432.

\subsection{Datos}

Los datos para este estudio fueron extraídos de entrevistas sociolingüísticas a 21 hablantes. El corpus de Iquitos consta de 100.466 palabras, y el de las comunidades de 83.237 , lo que da un total de 183.703 palabras. Para identificar construcciones de posesión nominal, se implementó un concepto estructural de posesión, independientemente del detalle semántico transmitido por la construcción. Es decir, el término posesión denota aquí una gama de significados, incluyendo relaciones de propiedad (ej. su casa), relaciones de parentesco (ej. su hermano), relaciones de todo-parte (ej. su mano), asociaciones (ej. su universidad), entre otros (cfr. Heine, 1997; Aikhenvald y Dixon, 2013).

Se identificaron y extrajeron manualmente todas las frases nominales con la estructura [PRO N], donde PRO es el pronombre posesivo de tercera persona, pero se excluyeron las expresiones idiomáticas ${ }^{7}$. Este procedimiento generó un 
total de 905 instancias de [PRO N], incluyendo construcciones de concordancia singular, [su N] (2); construcciones de concordancia plural, [sus Ns] (3), y la construcción innovadora con discordancia plural/singular, [sus N] (4).

los monos suben a su barbacoa sí, sus chacras se inundaron

ahí están los niños con sus regalo [su N]

[sus Ns]

[sus N]

Sin embargo, solo el 22\% (209/905) de estas instancias corresponden a situaciones comunicativas con poseedores múltiples, como en los ejemplos (2)(4). En el ejemplo (2), el pronombre su hace referencia a los monos; en (3), sus se refiere a varios comuneros, y en (4), sus a varios niños. Como el foco de este estudio son situaciones en las que el pronombre hace referencia a más de un poseedor, el total de instancias a ser analizadas será entonces 209. Las frecuencias de cada tipo de concordancia se muestran en la tabla 2.

Tabla 2. Construcciones posesivas con poseedores múltiples

\begin{tabular}{|l|c|c|}
\hline & $\mathrm{n}{ }^{\circ}$ & $\%$ \\
\hline Concordancia singular & 82 & 39 \\
\hline Concordancia plural & 75 & 36 \\
\hline Construcción innovadora & 52 & 25 \\
\hline Total & 209 & 100 \\
\hline
\end{tabular}

Elaboración propia.

Un punto notable en la tabla 2 es que, a pesar de reflejar una ruptura significativa de los patrones canónicos de concordancia, la construcción innovadora aparece en $25 \%$ de las instancias donde la alternancia es posible. Al considerar las frecuencias por variable, no hay una diferencia estadística entre las construcciones de concordancia singular y concordancia plural. Por ello, en la presentación de los resultados en $\S 4$, estas han sido colapsadas en la categoría construcción conservadora, y a la discordante nos referimos como construcción innovadora. 


\subsection{Superposición de construcciones}

Este estudio explora las áreas donde los hablantes pueden optar entre la concordancia y la discordancia (un contexto variable, en el sentido de Tagliamonte, 2011, p. 10). En el español canónico, el número del poseído determina el número del pronombre posesivo, independientemente del poseedor. Por lo tanto, se espera que los poseídos que hacen referencia a múltiples entidades sean codificados categóricamente por construcciones de concordancia plural y los poseídos únicos por construcciones de concordancia singular. En el EAP esto no siempre ocurre así. Sustantivos en singular pueden hacer referencia a un elemento poseído, como en (5); o múltiples poseídos, como en (6) y (7). En (6) se habla de que los candidatos a alcalde y a regidores colaboraron para adquirir botes para su campaña política; en (7) se habla de que cada anciano camina con su bastón.

\section{les ha hecho sus colegio}

\section{los candidatos compran sus bote}

Como resultado, una expresión dada como sus canoa podría ser interpretada como una sola canoa poseída por múltiples propietarios, varias canoas poseídas por un colectivo, o varios individuos, cada uno de los cuales posee una canoa (volveremos sobre este punto en \$3.4.4). En otras palabras, la construcción innovadora compite con la construcción de concordancia singular y la construcción de concordancia plural como se muestra en la figura 1. El área de superposición que se investiga en este artículo está indicada en rojo.

Figura 1: Superposición de construcciones

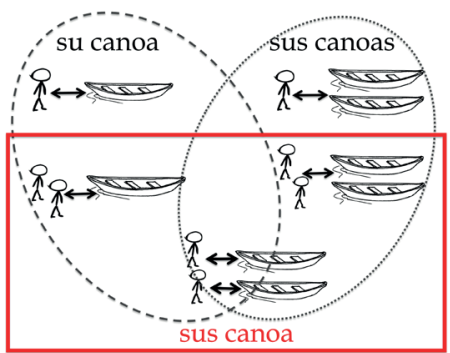

Elaboración propia. 


\subsection{Variables incluidas en este estudio}

Las variables sociales incluidas en este estudio son género del hablante (mujer/ hombre), y lugar de origen (comunidad/ciudad de Iquitos) ${ }^{8}$. En cuanto a las variables lingüísticas, consideramos los hallazgos de estudios tipológicos que han demostrado que la expresión lingüística de la posesión es a menudo condicionada por las propiedades semánticas de las entidades involucradas (Nichols y Bickel, 2011). Entre los parámetros relevantes, tenemos animacidad del poseedor y el poseído, la cuantificación/individuación del poseído y los subtipos de relaciones semánticas expresadas a través de la construcción [PRO N].

\subsubsection{Animacidad}

La animacidad es una característica inherente a una entidad. La jerarquía de animacidad consiste en un conjunto de categorías mutuamente exclusivas. Las entidades pueden clasificarse en diferentes categorías según su capacidad de comportamiento en relación con los humanos: humano, animado, inanimado. La idea es que la jerarquía de animación ordena a las entidades en una escala de relativa "prominencia". Esta propiedad semántica tiene un papel central en un sinnúmero de fenómenos morfosintácticos en las lenguas del mundo, incluyendo: los sistemas de caso, el orden de constituyente, la ergatividad escindida (Comrie, 1989; Croft, 1990), la indexación de argumentos en el verbo, las construcciones directas e inversas, la posesión externa (Givón, 2001), la distribución de los clíticos (Myhill, 1989), la marcación diferencial de objeto (Woolford, 1999), entre otras áreas de la gramática.

El rol de la animacidad en la expresión de la posesión está bastante establecido. En inglés, por ejemplo, la variación entre genitivo prenominal y genitivo posnominal (ej. The boy's toy versus The toy of the boy 'el juguete del niño') depende primariamente de la animacidad del poseedor: el genitivo prenominal desfavorece fuertemente poseedores inanimados (Rosenbach, 2008; Zaenen et al., 2004). Resultados paralelos han sido establecidos en diecisiete idiomas de las familias románica, germánica y eslava (O’Connor, Mailing y Skarabela, 2013). 
El rol de la animacidad en la gramática del español es claro; por ejemplo, en las construcciones de posesión externa, aquellas que subrayan la prominencia pragmática del poseedor (Givón, 2001; Myhill, 1989). Evidencias empíricas indican que cuando el poseedor se expresa dentro del sintagma nominal (ej. el perro mordió mi pie), el elemento resaltado es el poseído. En contraste, cuando el poseedor se expresa como un pronombre dativo fuera del sintagma nominal (ej. el perro me mordió el pie), el elemento resaltado es el poseedor (Winters, 2006, p. 157).

Más allá del poseedor, las propiedades semánticas del elemento poseído también tienen un papel probado en la expresión lingüística de la posesión. Las lenguas suelen tener diferentes formas de codificar las relaciones de posesión dependiendo de las propiedades semánticas de la entidad poseída. Por ejemplo, en una muestra de 243 lenguas, Nichols y Bickel (2013) encontraron que el 49\% de ellas posee lo que se conoce como clasificación posesiva; es decir, dichas lenguas emplean construcciones distintas dependiendo de la clase léxica a la que pertenezca el sustantivo poseído.

Basándonos en estos hallazgos, en el presente estudio examinamos las propiedades semánticas tanto del poseedor como del poseído. En tal sentido, se adaptó la jerarquía de animacidad propuesta por Zaenen et al. (2004). Esta jerarquía consta de diez categorías: humanos, organizaciones, animales, entidades automatizadas (computadoras, robots), vehículos, lugar, tiempo, entidades concretas, no-concretas y categoría mixta. Las categorías relevantes para dar cuenta de las entidades poseídas en nuestro corpus son cuatro: (i) humano, (ii) animado (animales y plantas), (iii) inanimado y (iv) abstracto; nótese que no se identificaron poseedores ni inanimados ni abstractos en la muestra. Algunos ejemplos se proporcionan a continuación:

(8) poseedor humano: todos tienen que acarrear sus agua [sus N]

(9) poseedor animado: los monos suben a su barbacoa [su N]

(10) poseído humano: que no fastidien, yo no soy sus nana [sus N] 
(11) poseído animado: ahora crían pues sus paiche [sus N]

(12) poseído inanimado: nos dejan regalando sus mosquiteros [sus Ns]

(13) poseído abstracto: nunca en sus vida se han preocupado [sus N]

\subsubsection{Cuantificación}

El español, como muchas otras lenguas, tiene subtipos de sustantivos dependiendo de su capacidad de ser cuantificados. Como se indicó anteriormente, la cuantificación del poseedor es decisiva en la aparición de la discordancia; por lo tanto, este estudio examina de manera exclusiva aquellos contextos que involucran claramente a varios poseedores. Sin embargo, las posibilidades de cuantificación del poseído son varias. La primera oposición considerada aquí es entre sustantivos contables versus incontables. Los primeros pueden ser cuantificados por números (ej. tres canoas), los segundos se refieren a sustancias o cualidades, no pueden tomar la marca de plural pero pueden ser cuantificados por expresiones (ej. un vaso de agua). Los sustantivos colectivos en su forma gramatical singular hacen referencia a varias entidades (ej. gente). Ejemplos que ilustran la codificación empleada en este estudio se ofrecen en (14) a (17).

(14) poseído único: hacen sus trampa [sus N]

(15) poseído múltiple: llevan calamina a sus casas [sus Ns]

(16) poseído colectivo: los candidatos buscan su gente [su N]

(17) poseído incontable: todos tienen que acarrear sus agua [sus N]

\subsubsection{Significados asociados a la posesión}

Existen varias tipologías de la posesión. Heine propone siete subtipos semánticos de posesión: física, temporal, permanente, inalienable, abstracta, inanimada inalienable, e inanimada (para detalles y ejemplos, véase Heine, 1997, pp. 3437). Además, este autor sostiene que la noción posesiva prototípica implica los siguientes rasgos: (i) un poseedor humano, (ii) un poseído concreto, (iii) un poseedor que tiene el derecho de usar al poseído, (iv) proximidad espacial entre poseedor y poseído, y (v) ningún límite temporal en la relación posesiva 
(Heine, 1997, p. 39). Por su parte, Aikhenvald (2003, pp. 3-5) plantea que algunos significados son centrales a la posesión y otros son más bien periféricos. En el primer grupo, tenemos: propiedad (ownership), parentesco, y relaciones partetodo. En el segundo grupo, se encuentran: asociación, orientación, y posesión metafórica. Ambas propuestas guían nuestra aproximación a los subtipos de posesión. Sin embargo, para evaluar de manera sistemática si la construcción innovadora está asociada con algún subtipo semántico/pragmático de posesión, hemos implementado la propuesta de Aikhenvald. Los siguientes ejemplos muestran nuestra codificación:

(18) propiedad: solo traían sus machete [sus N]

(19) parentesco: no le dan nada a sus hijos [sus Ns]

(20) parte-todo: a toditas las casas le quitan su techo [su N]

(21) asociación: ahí les hace sus colegio [sus N]

(22) posesión metafórica: no olvidan sus conocimientos [sus Ns]

\subsubsection{Posesión colectiva versus distributiva}

Un aspecto ampliamente estudiado en lingüística es la cuantificación de eventos. Existe una distinción básica entre eventos colectivos y eventos distributivos (Kemmer, 1993, p. 99). En los eventos colectivos, el referente del sujeto es un grupo, el cual se entiende como un conjunto de individuos que realizan la acción de forma conjunta. En los eventos distributivos existen también varios sujetos, pero cada uno realiza la acción de forma independiente. El colectivo difiere del distributivo en la presentación del evento como algo unitario. Por ejemplo, la oración "Los invitados abandonaron la fiesta" puede recibir ambas lecturas. En la interpretación colectiva, los invitados salieron de la fiesta en grupo de tal manera que el salir se presenta como un evento único; en la distributiva, dejaron la fiesta uno por uno, y en este caso estaríamos ante varios eventos de salir.

La distinción colectiva versus la distributiva se aplica también a la posesión (Mithun 1999, p. 89; 2001). En la posesión colectiva, el elemento poseído es propiedad de un grupo, mientras que en la distributiva el elemento poseído es distribuido entre los miembros de un colectivo. Muchas lenguas tienen 
estrategias gramaticales para codificar la distinción colectiva/distributiva. Por ejemplo, varios idiomas amerindios, incluido el quechua, poseen marcadores distributivos asociados a sustantivos, adjetivos, numerales, pronombres posesivos y, más comúnmente, a verbos (Mithun, 1999, p. 89).

En Vallejos (2014, p. 442), se sugiere que la construcción innovadora parecería estar desarrollando una función dedicada a la posesión distributiva. Para evaluar esta hipótesis, cada instancia del corpus fue codificada por esta variable, como se muestra en los siguientes ejemplos:

(23) distributiva: ahi están los niños con sus regalo

[sus N]

(24) colectiva: los monos suben a su barbacoa [su N]

\section{Resultados}

En esta sección discutimos la frecuencia de las construcciones posesivas para determinar si existen diferencias significativas en cuanto a su uso (utilizando la prueba chi-cuadrado). Para discutir las frecuencias de uso por variable, los resultados de las construcciones canónicas - concordancia singulary concordancia plural - han sido colapsadas en la categoría construcción conservadora, la que será contrastada con la construcción innovadora. La discusión comienza con las variables sociales, en $\S 4.1$ y luego procede a las variables lingüísticas, en $\S 4.2, \S 4.3$ y $§ 4.4$. Finalmente, el análisis de regresión logística (utilizando Rbrul) para calcular la significancia de cada variable independiente en el uso de la construcción innovadora es presentado en $\S 4.5$.

\subsection{Variables sociales}

Los resultados del rol de las variables sociales en la alternancia morfosintáctica se presentan en las figuras 2 y 3 . La figura 2 muestra que el uso de la construcción innovadora no es significativamente diferente en las comunidades y la ciudad de Iquitos. En la comunidad, la discordancia se produce en el 25\% (34/135) de los contextos con poseedores múltiples, mientras que en la ciudad aparece en el $24 \%(18 / 74)$ de casos. Estos resultados sugieren que el uso de la construcción innovadora trasciende la variable lugar de origen del hablante. 
Figura 2. Ciudad versus comunidad

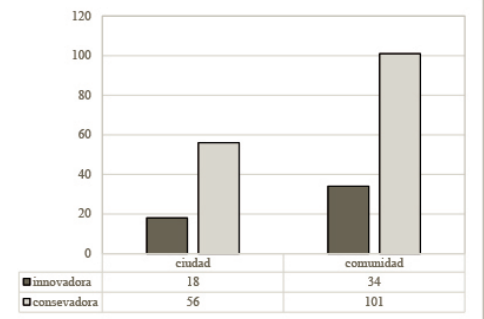

Elaboración propia.
Figura 3. Género del hablante

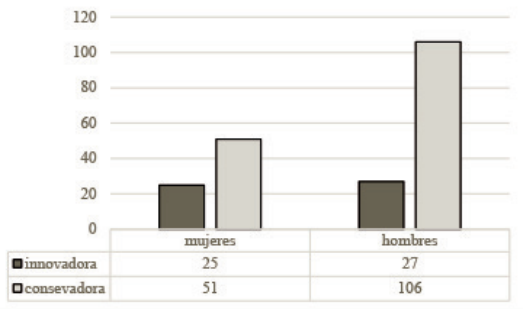

Elaboración propia.

La figura 3 presenta el rol del género del hablante. Aquí notamos que mientras las mujeres usan la construcción innovadora en 33\% (25/76) de los casos con poseedores múltiples, los hombres lo hacen en 20\% (27/133) de los casos. Esta diferencia es significativa $\left(\chi^{2}(1)=4,10, \mathrm{p}=, 043\right)$. Es decir, las mujeres tienden a usar con mayor frecuencia la discordancia.

\subsection{Animacidad del poseedor y el poseído}

La figura 4 revela una obvia asimetría con respecto a la animacidad del poseedor. Primero, el 87\% (181/209) de los poseedores en nuestra muestra es humano; el $10 \%(21 / 209)$ es animado. En contraste, la presencia de poseedores inanimados es minúscula $(3 \%, 7 / 209)$. Segundo, la construcción innovadora no se usa cuando el poseedor es inanimado. En el 92\% (48/52) de casos con patrón discordante, el poseedor es humano, y solo en $8 \%$ (4/52), el poseedor es animado.

Figura 4. Animacidad del poseedor

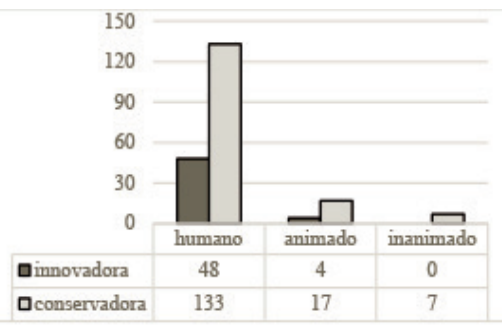

Elaboración propia.
Figura 5. Animacidad del poseído

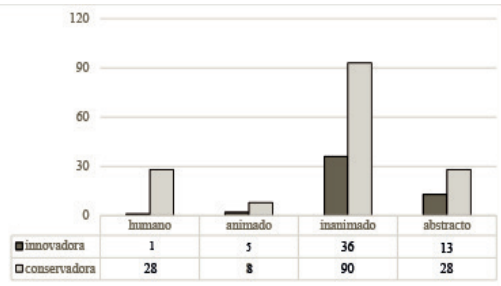

Elaboración propia. 
En la figura 5 vemos que el referente del poseído es más diverso en cuanto a animacidad, aunque los resultados son casi opuestos a los del poseedor. El 60\% (126/209) de los poseídos es inanimado, el 20\% (41/209) es abstracto, el $14 \%$ (29/209) es humano y el 6\% (13/209) es animado. Un punto interesante es que la construcción innovadora es usada con entidades que se ubican en diferentes puntos de la escala de animacidad, pero su aparición con poseídos humanos $(2 \%, 1 / 55)$ es ciertamente mínima. La combinación de poseedor/poseído más frecuente en la muestra involucra entidades en ambos extremos de la jerarquía de animacidad: poseedor humano/poseído inanimado (55\%, 107/209), y en estos contextos la construcción innovadora es usada en el 30\% (32/107) de los casos. Estos resultados fortalecen la hipótesis de que la animacidad es un factor clave para el uso de la construcción innovadora.

\subsection{Cuantificación}

La figura 6 muestra que, en el 62\% (129/209) de las instancias, el poseído hace referencia a varias entidades, y en el 31\% (65/209), el poseído es una entidad única. El porcentaje de poseídos expresado en sustantivos incontables o colectivos es mínimo.

Figura 6. Cuantificación del poseído

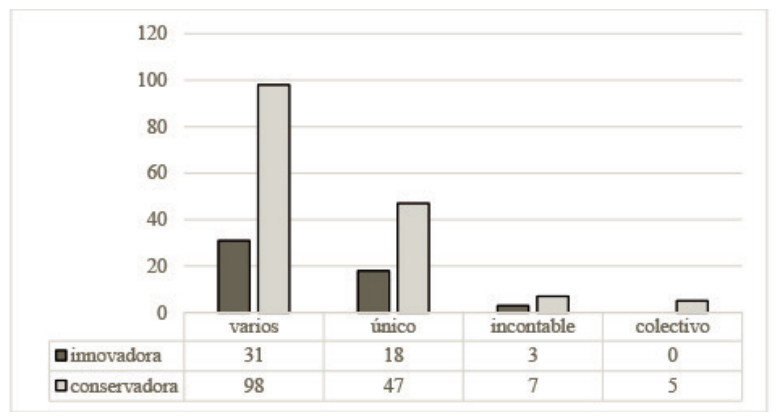

Elaboración propia.

Sin embargo, el uso de construcciones innovadoras al interior de cada categoría es bastante similar: en la categoría varios es el 24\% (31/129), en único $28 \%$ (18/65) y en incontable 30\% (3/10). En efecto, un análisis de chicuadrado revela que no existe una diferencia significativa en cuanto al uso de la 
construcción conservadora e innovadora entre estas tres categorías. La categoría que tiene una distribución diferente es colectivo, $0 \%(0 / 5)$, pero no sería prudente sacar conclusiones definitivas dado que el número de instancias de sustantivos colectivos en la muestra es pequeño.

\subsection{Tipos de relaciones posesivas}

La figura 7 revela que hay una escala de frecuencias en cuanto a los tipos de posesión, desde propiedad, en el extremo más frecuente $(43 \%, 89 / 209)$, hasta relaciones parte-todo $(7 \%, 15 / 209)$, en el lado menos frecuente. Sin embargo, al interior de cada categoría, el uso del patrón innovador es bastante similar. En la categoría propiedad, el 28\% (25/89) de casos usa la construcción discordante; en asociación, el 22\% (11/51); en posesión metafórica, el 29\% (8/28); en parentesco, el 15\% (4/22); y en parte-todo, el 27\% (4/15). La prueba chi-cuadrado revela que no hay una diferencia significativa en el uso de ambas construcciones. En resumen, la construcción innovadora no está especializada en ningún subtipo de posesión.

Figura 7. Tipos de relaciones posesivas

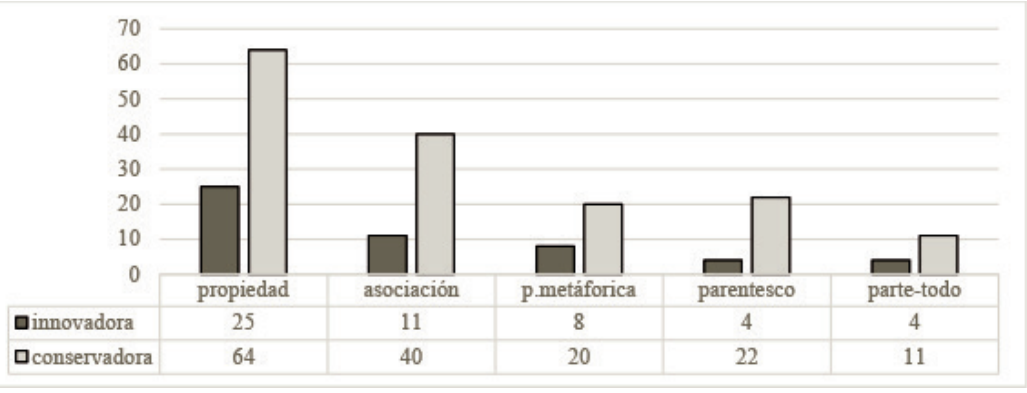

Elaboración propia.

Los resultados sobre la posesión colectiva y la posesión distributiva se ofrecen en la figura 8. En el 75\% (157/209) de los casos, la interpretación es distributiva, y solo en el 25\% (52/209) es colectiva. Sin embargo, al interior de cada categoría, el porcentaje de uso de la construcción innovadora es bastante similar, 26\% (16/52) en la distributiva, y 22\% (59/157) en la colectiva. La prueba chi-cuadrado confirma estas observaciones: no existe una diferencia significativa en la distribución de las construcciones en ambas categorías. 
Figura 8. Posesión colectiva versus distributiva

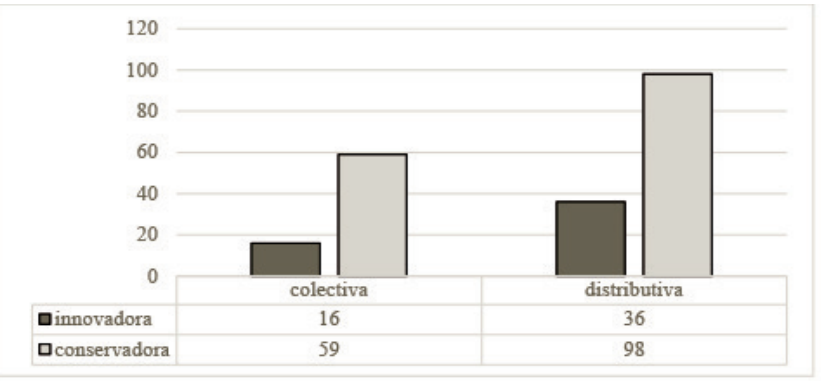

Elaboración propia.

4.5 Variables que influyen de manera significativa en el uso del patrón innovador Para identificar qué variables independientes influyen de manera significativa en la variable dependiente, se ejecutó un análisis de regresión logística (mixed model) utilizando RBrul (Johnson, 2009). La variable dependiente del modelo es la construcción innovadora, y las independientes son: género, lugar, animacidad del poseedor, animacidad del poseído, cuantificación del poseído, subtipos de posesión y relación distributiva/colectiva. La variable hablante fue incluida como efecto aleatorio. Un resumen de los factores significativos que arroja Rbrul se presenta en la figura 9. Los valores positivos favorecen la aparición de la construcción innovadora, los negativos la desfavorecen. La longitud de la barra es proporcional a la fuerza del efecto. Los factores que resultaron significativos son género del hablante $(\mathrm{p}=, 0455)$ y la animacidad del poseído $(\mathrm{p}=, 00948)$.

Figura 9. Variables significativas (modelo de regresión mixta)

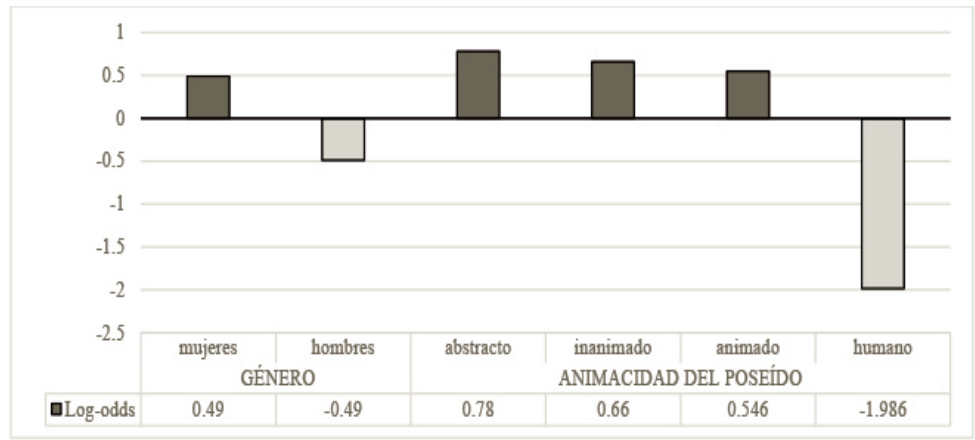

Elaboración propia. 
El análisis de regresión confirma las observaciones hechas al analizar las frecuencias de cada variable de manera independiente. Las mujeres son ligeramente más propensas a usar el patrón innovador que los hombres. En cuanto a la animacidad del poseído, es ligeramente más probable que se use el patrón innovador si este es abstracto o inanimado, y es indiscutible que los poseídos humanos claramente desfavorecen la discordancia. Nótese que la animacidad del poseedor no resultó significativa porque en la muestra la gran mayoría son humanos, y no se identificaron poseedores inanimados. Es decir, la animacidad del poseedor es prácticamente categórica, no variable. El hecho de que, por un lado, los poseedores sean generalmente humanos, y de que, por otro lado, los poseídos humanos desfavorezcan la discordancia confirma que la animacidad es la variable más importante para el surgimiento del patrón innovador.

\section{Discusión}

¿Cuál es la frecuencia de la construcción innovadora en relación con las conservadoras? La construcción innovadora constituye el 6\% (52/905) de todas las construcciones posesivas [PRO N] en la base de datos. Sin embargo, cuando nos enfocamos exclusivamente en aquellos contextos variables donde los poseedores son múltiples (véase tabla 2), el porcentaje se eleva a 25\% (52/209). Esta cifra no puede subestimarse, pues revela la existencia de un patrón morfosintáctico en proceso de generalización.

¿Qué factores sociales (des)favorecen el uso de la construcción innovadora? El patrón discordante está bastante extendido y trasciende edad, género, ocupación, y comunidad de origen. Nótese que los hablantes entrevistados pertenecen a diferentes grupos sociales y las observaciones se hicieron en una muestra balanceada por comunidad de origen, género y grupos generacionales. En Vallejos (en evaluación), se encontró que la discordancia era ligeramente más alta en las comunidades rurales; no obstante, este estudio revela que, cuando la atención se centra en contextos con múltiples poseedores, no existe una diferencia significativa entre el uso del patrón innovador en las comunidades y la ciudad. Los resultados con relación a género son consistentes, el uso de la discordancia es 
ligeramente más alto entre las mujeres que entre los hombres. Este factor resultó significativo en el análisis de regresión.

¿Qué factores lingüísticos (des)favorecen el uso de la construcción innovadora? En español canónico el sustantivo principal determina el número y el género de todos sus determinantes y modificadores, por lo tanto, la falta de concordancia entre el pronombre posesivo y su núcleo es excepcional. Además de la cabeza sintáctica (el poseído), un nuevo candidato parece estar emergiendo como un referente potencial para el pronombre posesivo: su anáfora (el poseedor). Dada las abundantes evidencias del rol de la animacidad en las diferentes áreas de la gramática, la primera hipótesis que se evalúa aquí es si la animacidad de los referentes del poseedor y el poseído influyen en el uso del patrón discordante. Los resultados indican que el patrón innovador no ocurre con los poseedores al azar, sino que impone restricciones semánticas de variabilidad sobre ellos. La primera restricción conocida es la cuantificación de las entidades poseedores (Vallejos, en evaluación). El uso de la construcción innovadora aumenta de manera significativa si los poseedores son múltiples.

La segunda restricción se refiere a la animacidad de los referentes. Cuando el poseedor y el poseído se encuentran en diferentes niveles en la jerarquía de animacidad, el más alto en la jerarquía gana el control de la concordancia de número, lo que da como resultado la "supuesta discordancia" al interior de la frase nominal. Sin embargo, cuando ambos están en el mismo nivel (por ejemplo, cuando ambos son humanos), el control de la concordancia la gana el poseído, el núcleo del sintagma, siguiendo patrones canónicos. Esto no es extraño porque, primero, según la jerarquía de animacidad los humanos son más prominentes que las entidades inanimadas (Comrie, 1989; Givón, 2001; entre otros); y, segundo, la posesión prototípica involucra un poseedor humano y un poseído concreto, inanimado (Heine, 1997, p. 39). En efecto, como se muestra en la figura 4, el tipo de poseedor más frecuente es humano (87\%). La figura 5 muestra que el tipo de poseído más frecuente es inanimado $(60 \%)$. La combinación de poseedor/ poseído más frecuente en la muestra involucra entidades en ambos extremos de la jerarquía de animacidad: poseedor humano/poseído inanimado (55\%). En dichos 
contextos, los poseedores dominan al poseído en función de la prominencia. En suma, los resultados de este estudio se suman a las evidencias del rol de la animacidad en la expresión lingüística de la posesión, así como en otros dominios funcionales (Rosenbach, 2008; Zaenen et al., 2004; Winters, 2006; O’Connor, Mailing y Skarabela, 2013).

¿La construcción innovadora está asociada con algún subtipo de posesión? El patrón discordante no invoca una relación semántico/pragmática en particular. Los resultados revelan que si bien hay una escala de frecuencias de los diferentes tipos de posesión, desde propiedad en el extremo más frecuente (43\%), hasta relaciones parte-todo (7\%) en el lado menos frecuente (figura 7), el uso del patrón innovador en cada categoría es bastante similar. La evaluación de la hipótesis sobre una posible especialización del patrón emergente para distinguir entre posesión colectiva y posesión distributiva revela que tal especialización no existe (figura 8). En general, la interpretación más común es la distributiva (75\%), pero el uso de la discordancia dentro de cada categoría no es significativamente diferente. En suma, el patrón innovador se usa para los diferentes tipos de posesión y es inmune a la distinción colectiva/atributiva. Así, las interpretaciones relacionales (figura 1) se derivan del contexto o del razonamiento pragmático.

¿Cómo surge el patrón innovador? Existe amplia evidencia de que las presiones pragmáticas y cognitivas influyen en el uso y adquisición del lenguaje (Bybee y Hopper, 2001; Diessel, 2007). Por un lado, existen limitaciones de procesamiento que favorecen la minimización del esfuerzo de codificación (economía), así como la minimización del esfuerzo de decodificación (distintividad). Por otro lado, hay preferencias conceptuales-pragmáticas de los hablantes hacia ciertos referentes en el uso del lenguaje (Haspelmath, 2008). Como señala Haspelmath, “[...] los hablantes no tienen la intención de crear gramáticas bien diseñadas, sino que se comportan de manera práctica y racional al seleccionar variantes disponibles y al crear nuevas variantes" (2008, p. 205; traducción propia). La innovación gramatical que se reporta aquí es un ejemplo más de cómo los hablantes prestan atención a los referentes más prominentes. Pero si la mayoría de los poseedores son altos en animacidad, la pregunta es 
por qué el surgimiento de la construcción innovadora parece lento. Evidencias empíricas han demostrado que la frecuencia tiene un impacto en el surgimiento de categorías lingüísticas (Bybee y Hopper, 2001). Existen importantes distribuciones asimétricas en la base de datos que podrían explicar esta situación. Contextos con múltiples poseedores constituyen solo el 22\% (209/905) del total de construcciones posesivas [PRO N]. Hay entonces una presión analógica sustancial para retener la concordancia canónica en los contextos con un solo poseedor, al ser estos mucho más frecuentes en el discurso. También llama la atención el hecho de que solo el $25 \%$ de instancias donde los contextos son favorables sean expresados a través de la construcción innovadora (tabla 2). Sabemos que los pronombres suelen tener referentes en foco, activados o altamente accesibles (Ariel, 2001; O’Connor, Mailing y Skarabela 2013). La evaluación del papel que juega el estado pragmático del poseedor en la alternancia morfosintáctica es entonces una línea de investigación prometedora (Vallejos, 2017).

Finalmente, ¿por qué este patrón no ha surgido en otras variedades de español? Como se indica en $\$ 3.1$, el español en la región Loreto está en contacto con muchas lenguas indígenas locales. En efecto, aun cuando en las comunidades de este estudio la lengua indígena kukama ha sido desplazada por el español en la comunicación diaria, todavía existen hablantes de esta lengua y, además, hay diversas iniciativas de revitalización en marcha. Podría considerarse la posibilidad de un efecto de sustrato kukama dado que la construcción de la posesión nominal en esa lengua es exactamente igual al patrón innovador en el EAP(Vallejos, 2016). Además, los hallazgos reportados aquí son consistentes con otras características no canónicas que son más frecuentes en las comunidades kukamas que en la ciudad de Iquitos (Vallejos, 2014; Vallejos y Koops, 2016). Sin embargo, puesto que en la actualidad el porcentaje de uso de la discordancia es similar en las comunidades y en la ciudad, cualquier posible efecto de contacto tendría que ser corroborado cuidadosamente con datos correspondientes a diferentes períodos históricos que nos ayuden a desentrañar la formación del español en la Amazonía de Perú. 


\section{Conclusiones}

El presente estudio proporciona evidencias contundentes sobre la existencia de una construcción morfosintáctica innovadora en el español amazónico peruano, y nos plantea la disyuntiva de si en verdad cabe hablar de "discordancia" en los contextos discutidos aquí. Dentro del dominio de posesión nominal, hay un patrón en el que el pronombre posesivo concuerda en número con la entidad a la que hace referencia anafórica, no con su núcleo sintáctico, como sucede en el español canónico. Este estudio demuestra que el uso de la construcción innovadora está generalizada en todo el espectro social tanto de comunidades rurales como en centros urbanos, y trasciende las diferentes generaciones, grupos sociales y ocupaciones.

Los resultados sugieren, sin embargo, que este patrón es ligeramente más común entre las mujeres. La construcción innovadora es más probable de ser usada cuando los poseedores son humanos y los poseídos no-humanos. Al parecer, las motivaciones para el surgimiento de esta nueva estructura serían fuerzas cognitivas generales. Primero, las entidades ubicadas en puntos más altos en la jerarquía de la animacidad son más prominentes que aquellas en el extremo inferior de la jerarquía; y, segundo, la relación de posesión prototípica, y más común en el corpus, involucra a poseedores humanos y poseídos inanimados. Este estudio proporciona evidencias claras de que el lenguaje es un fenómeno emergente y dinámico. Si el uso de la forma innovadora es una tendencia creciente, como parece, estamos asistiendo a una fase incipiente de gramaticalización.

\section{Notas}

1 Deseo expresar mi agradecimiento profundo a cada uno de los hablantes de español amazónico entrevistados para este estudio. Agradezco a Jaime Peña así y a los evaluadores anónimos de la revista por sus comentarios editoriales y analíticos. Los datos presentados aquí se recogieron en múltiples trabajos de campo financiados por el Research Allocations Committee y el Latin American y Iberian Institute, ambos de la Universidad de Nuevo México.

2 La Amazonía peruana comprende las regiones de Loreto, Ucayali, Madre de Dios y San Martín, siendo Loreto la región geográficamente más amplia de todas. 
3 Por ejemplo, Mithun (2001, pp. 288-294) reporta que en Lakhota (Sioua), Kathlamet (Chinooka) y Mohawk (Iroquoia), la afectividad del poseedor tiene un rol en la elección entre las construcción atributivas y las clausales. La construcción clausal se utiliza cuando el poseedor es el participante más afectado.

4 Se ha documentado una serie de características lingüísticas para el EAP. Por ejemplo, entre los rasgos fonológicos figuran la permutación entre [f] y $[\mathrm{x}]$, y la fortificación de palatales [y] y [ $\lambda]$ ) (Escobar, 1978; Ramírez, 2003). Los rasgos morfosintácticos más sobresalientes son la posesión doble, la preposición del genitivo, el leísmo y el loísmo (Escobar, 1978; Caravedo, 1997), la doble marcación de objetos directos, falta de concordancia en las construcciones con cópula (Barraza de la Cruz 1998). Además, el EAP posee una estructura prosódica particular (Escobar, 1978; García, 2011; Vallejos y Koops 2016). Es importante destacar que la reducción y aspiración de /s/ no son procesos característicos de esta variedad, aunque la supresión de diferentes tipos de segmentos, e incluso sílabas, es frecuente (Vallejos y Koops 2016).

5 Ramírez (2003, p. 15) explica que los datos de su libro provienen de él mismo, como hablante nativo de la variedad de Moyobamba (San Martín); aunque, como indica, en algún momento tuvo acceso a hablantes de Loreto. Si en San Martín la discordancia de número se extiende a los pronombres de primera y segunda persona, la explicación para la discordancia no serían referentes anafóricos múltiples, como sucede en Loreto.

6 Casas Navarro (2015) reporta que el dequeísmo es un proceso de cambio sintáctico extendido entre jóvenes peruanos de 15 a 25 años de edad; sin embargo, la educación formal en gramática normativa es una presión que se opone significativamente a este cambio.

7 Una estrategia empleada para identificar las instancias a excluir fue la capacidad de ser parafraseadas como [ $\mathrm{N}$ de referente $\mathrm{X}]$. Por ejemplo: su casa puede parafrasearse como la casa de él/ella/ellos/ellas. Algunos ejemplos de expresiones que fueron excluidas son: (a) el bote estaba hasta sus últimas; (b) ha puesto su granito de arena, (c) espero que pongan de su parte.

8 Por razones de espacio no incluimos la variable edad del hablante. Basta señalar que el patrón innovador es usado por todos los grupos generacionales; es decir, edad no juega un rol en la alternancia, confirmando los resultados presentados en Vallejos (en evaluación).

\section{Referencias bibliográficas}

Aikhenvald, A. y Dixon, R. (2013). Possession and Ownership: a cross-linguistic typology. Oxford: Oxford University Press.

Ariel, M. (2001). Accessibility Theory: An Overview. En T. Sanders, J. Schilperoord, y W. Spooren (Eds.), Text Representation: Linguistic and Psycholinguistic Aspects (29-87). Ámsterdam/Filadelfia: John Benjamins. 
Barraza de la Cruz, Y. (1998). Apuntes sobre gramática del castellano de Iquitos. (Tesis para optar el grado académico de Magister en Lingüística). Universidad Nacional Mayor de San Marcos. Facultad de Letras y Ciencias Humanas, Lima, Perú.

Barras, C., Geoffrois, E., Wu, Z., y Liberman, M. (2000). Transcriber: development and use of a tool for assisting speech corpora production. Speech Communication (special issue on Speech Annotation and Corpus Tools), 33(1-2), 5-22. doi: 10.1016/S0167-6393(00)00067-4.

Bybee, J. y Hopper, P. (Eds.). (2001). Frequency and the emergence of linguistic structure. Ámsterdam: John Benjamins. doi: 10.1075/tsl.45.

Caravedo, R. (1997). Los pronombres objeto en un corpus del español amazónico peruano. Anuario de Letras, 35, 131-155. doi: 10.19130/iifl. adel.35.0.1997.816.

Casas Navarro, R. (2015). El dequeísmo: ¿un cambio en progreso de la sintaxis castellana? Letras, 86(124), 289-310. Recuperado de http://revista. letras.unmsm.edu.pe/index.php/le/article/view/305/300.

Comrie, B. (1989). Language Universals and Linguistic Typology. Chicago: The University of Chicago Press.

Croft, William. (1990). Typology and Universals. Cambridge: Cambridge University Press.

Diessel, H. (2007). Frequency effects in language acquisition, language use, and diachronic change. New Ideas in Psychology 25, 108-127. doi: 10.1016/j. newideapsych.2007.02.002.

Escobar, A. (1978). Variaciones sociolingüísticas del castellano en el Perú. Lima: Instituto de Estudios Peruanos.

García, M. (2011). The intonational patterns of the Peruvian Amazonian Spanish (PAS). Nueva York: State University of New York at Stony Brook. Recuperado de https://bit.ly/2Q5LtSZ.

Givón, T. (2001). Syntax, vols.1 y 2. Ámsterdam, Filadelfia: John Benjamins.

Haspelmath, M. (2008). Creating economical morphosyntactic patterns in language change. En J. Good (Ed.), Linguistic Universals and Language Change (185-214). Oxford: Oxford University Press. doi: 10.1093/ acprof:oso/9780199298495.001.0001. 
Heine, B. (1997). Possession: cognitive sources, forces, and grammaticalization. Cambridge: Cambridge University Press.

INEI-Instituto Nacional de Estadística e Informática. (2017). Censos Nacionales 2017: XII de Población, VII de Vivienda y III de Comunidades Indígenas. Recuperado de http://censos2017.inei.gob.pe/redatam/.

Johnson, D. (2009). Getting off the GoldVarb Standard: Introducing Rbrul for Mixed-Effects Variable Rule Analysis. Language and Linguistics Compass, 3(1), 359-383. doi: 10.1111/j.1749-818x.2008.00108.x

Kemmer, S. (1993). The Middle Voice. Ámsterdam, Filadelfia: John Benjamins. doi: $10.1075 /$ tsl.23.

Lipski, J. (1994). Latin American Spanish. Londres: Longmans.

Mithun, M. (2001). The difference a category makes in the expression of possession and inalienability. En M. Herslund, F. Soerensen, y I. Baron (Eds.), Dimensions of Possession (143-168). Ámsterdam: John Benjamins. doi: 10.1075/tsl.47.16mit.

Myhill, J. (1989). Variation in Spanish clitic climbing. En T. J. Walsh (Ed.), Synchronic and diachronic approaches to linguistic variation and change (227-250). Washington, D. C.: Georgetown University Press.

Nichols, J. y Bickel, B. (2013). Possessive Classification. En M S. Dryer y M. Haspelmath (Eds.), The World Atlas of Language Structures Online. Leipzig: Max Planck Institute for Evolutionary Anthropology. Recuperado de http://wals.info/chapter/59.

O’Connor, C., Mailing, J., y Skarabela, B. (2013). Nominal Categories and the Expression of Possession: A Cross-Linguistic Study of Probabilistic Tendencies and Categorical Constrains. En K. Börjars, D. Denison, y A. Scott (Eds.), Morphosyntactic Categories and the Expression of Possession (89-122). Ámsterdam, Filadelfia: John Benjamins.

Queixalós, F. (2009). Amazonía: Aspectos Generales. En Atlas sociolingüístico de pueblos indígenas en América Latina, editado por Inge Sichra (231244). Quito: AECID, FUNPROEIB Andes, UNICEF. Recuperado de https://www.unicef.org/honduras/tomo_1_atlas.pdf.

Ramírez, L. H. (2003). El español amazónico hablado en el Perú. Lima: Juan Gutemberg Editores. 
Rosenbach, A. (2008). Animacy and grammatical variation: Findings from English genitive variation. Lingua 118(2), 151-171. doi: 10.1016/j. lingua.2007.02.002.

Solís Fonseca, G. (2009). Perú Amazónico. En Atlas sociolingüístico de pueblos indígenas en América Latina, editado por Inge Sichra (302-332). Quito: AECID, FUNPROEIB Andes, UNICEF. Recuperado de https://www. unicef.org/honduras/tomo_1_atlas.pdf.

Stassen, L. (2009). Predicative possession. Oxford: Oxford University Press.

Tagliamonte, S. (2011). Variationist Sociolinguistics: Change, Observation, Interpretation. John Wiley y Sons.

Vallejos, R. (2014). Amazonian Spanish: Uncovering Variation and deconstructing Stereotypes. Spanish in Context 11(3), 425-453. doi: 10.1075/ sic.11.3.06val

Vallejos, R. (2016). A Grammar of Kukama-Kukamiria: A language from the Amazon. Leiden: Brill.

Vallejos, R. (2017). Effects of information structure in sentence-level grammar: innovative possession in Peruvian Amazonian Spanish. Presentado en la conferencia LASSO XLVI. Las Cruces, NM. 5-7 de octubre.

Vallejos, R. (en evaluación). Nominal possession in Peruvian Amazonian Spanish: the role of animacy in the emergence of grammar. En A. M. Escobar, R. Zariquiey, P. Valenzuela y M. Jara (Eds.), Spanish Diversity in the Amazon: Dialect and Language Contact Perspectives.

Vallejos, R. y Koops, C. (2016). A substrate account of Peruvian Amazonian Spanish Prosody. Presentado en $8^{\text {th }}$ International Workshop on Spanish Sociolinguistics, San Juan, Puerto Rico, EE. UU., 13-16 de abril.

Zaenen, A., Carletta, J., Garretson, G., Bresnan, J., Koontz-Garboden, A., Nikitina, T., O'Connor, M. C. y Wasow, T. (2004). Animacy encoding in English: why and how. En D. Bayron y B. Webber (Eds.). Proceedings of the Association for Computational Linguistics Workshop on Discourse Annotation (118-125). EE. UU.: East Stroudsburg.

Woolford, E. (1999). Animacy hierarchy effects on object agreement. En P. Kotey (Ed.), New Dimensions in African Linguistics and Languages (203-216). Nueva Jersey: Africa World Press. 